\title{
Learning-Based Distributionally Robust Model Predictive Control of Markovian Switching Systems with Guaranteed Stability and Recursive Feasibility
}

\author{
Mathijs Schuurmans and Panagiotis Patrinos
}

\begin{abstract}
We present a data-driven model predictive control scheme for chance-constrained Markovian switching systems with unknown switching probabilities. Using samples of the underlying Markov chain, ambiguity sets of transition probabilities are estimated which include the true conditional probability distributions with high probability. These sets are updated online and used to formulate a time-varying, risk-averse optimal control problem. We prove recursive feasibility of the resulting MPC scheme and show that the original chance constraints remain satisfied at every time step. Furthermore, we show that under sufficient decrease of the confidence levels, the resulting MPC scheme renders the closed-loop system mean-square stable with respect to the true-but-unknown distributions, while remaining less conservative than a fully robust approach.
\end{abstract}

\section{INTRODUCTION}

Stochasticity is inherent to processes emerging in a multitude of applications. Nevertheless, we are often required to control such systems, given the information that is available. Over the past decades, the decreasing cost of data acquisition, transmission and storage has caused a surge in research interest in data-driven approaches towards control. More recently, as the focus in research is gradually shifting towards real-life, safety-critical applications, there has been an increasing concern for safety guarantees of such datadriven methods, which are valid in a finite-data regime (see [1] for a recent survey).

In this paper, we focus on safe, learning-based control of Markov switching systems. Control of this class of systems is widely studied and has been used to model systems stemming from a wide range of applications [2]-[5].

Many of these approaches, however, require knowledge of the transition kernel governing the switching behavior of these systems. In practice, such information is typically not known. Recently, some work has been done to address this problem using data [6], [7]. However, relatively little attention has gone to providing a priory guarantees on stability and constraint satisfaction.

Leveraging the framework of risk-averse model predictive control [8]-[10] and its connection to distributionally robust

M. Schuurmans and P. Patrinos are with the Department of Electrical Engineering (ESAT-STADIUS), KU Leuven, Kasteelpark Arenberg 10, 3001 Leuven, Belgium. Email: \{mathijs.schuurmans, panos.patrinos\}@esat. kuleuven.be

This work was supported by: FWO projects: No. G086318N; No. G086518N; Fonds de la Recherche Scientifique - FNRS, the Fonds Wetenschappelijk Onderzoek-Vlaanderen under EOS Project No. 30468160 (SeLMA), Research Council KU Leuven C1 project No. C14/18/068 and the Ford-KU Leuven Research Alliance project No. KUL0023. optimization [11], we propose a learning-based distributionally robust model predictive control scheme, which is provably stable, recursively feasible and uses data gathered during operation to improve performance, rendering it less conservative than more traditional robust approaches [12]. Despite its growing popularity [10], [13]-[19], this approach has remained relatively unexplored for chance-constrained stochastic model predictive problems with ambiguity in the estimation of conditional distributions, and in particular for Markovian switching dynamics.

We summarize our contributions as follows. (i) We propose a general formulation for data-driven, distributionally robust model predictive control scheme for Markov switching systems with unknown transition probabilities, which is compatible with the recently developed framework of risk-averse model predictive control [8], [9]. The resulting closed-loop system satisfies the (chance) constraints of the original stochastic problem and allows for online improvement of performance based on observed data. (ii) We state the problem in terms of an augmented state vector of constant dimension, which summarizes the available information at every time. This idea, which is closely related to that of sufficient statistics [20, Ch. 5] and belief states in partially-observed Markov decision processes [21] allows us to formulate the optimal control problem as a dynamic programming recursion. (iii) Using techniques from dynamic programming, we provide sufficient conditions for recursive feasibility and mean-square stability of the data-driven model predictive control (MPC) scheme, with respect to the true-but-unknown probability distributions.

\section{A. Notation}

Let $\mathbb{N}$ denote the set of natural numbers and $\mathbb{N}_{>0}:=\mathbb{N} \backslash 0$. For two naturals $a, b \in \mathbb{N}, a \leq b$, we denote $\mathbb{N}_{[a, b]}:=\{n \in$ $\mathbb{N} \mid a \leq n \leq b\}$ and similarly, we introduce the shorthand $w_{[a, b]}:=\left(w_{t}\right)_{t=a}^{b}$ to denote a sequence of variables. We denote the extended real line by $\overline{\mathbb{R}}:=\mathbb{R} \cup\{ \pm \infty\}$ and the set of nonnegative (extended) real numbers by $\mathbb{R}_{+}$(and $\overline{\mathbb{R}}_{+}$). The cardinality of a (finite) set $W$ is denoted by $|W|$. Given a matrix $P \in \mathbb{R}^{n \times m}$, we denote its $(i, j)^{\prime}$ 'th element by $P_{i j}$ and its $i$ 'th row as $P_{i:} \in \mathbb{R}^{m}$. The $i$ 'th element of a vector $x$ is denoted $x^{i}$ to avoid confusion with time indices. $\operatorname{vec}(M)$ denotes the vertical concatenation of the columns of a matrix $M$. We denote the vector in $\mathbb{R}^{k}$ with all elements one as $\mathbf{1}_{k}:=(1)_{i=1}^{k}$ and the probability simplex of dimension $k$ as $\Delta_{k}:=\left\{p \in \mathbb{R}_{+}^{k} \mid p^{\top} \mathbf{1}_{k}=1\right\}$. We define the indicator 
function as $1_{x=y}:=1$ if $x=y$ and 0 otherwise. Similarly, the characteristic function $\delta_{\mathcal{X}}: \mathbb{R}^{n} \rightarrow \overline{\mathbb{R}}$ of a set $\mathcal{X} \in \mathbb{R}^{n}$ is defined by $\delta_{\mathcal{X}}(x)=0$ if $x \in \mathcal{X}$ and $\infty$ otherwise. The interior of a set $\mathcal{X}$ is denoted int $\mathcal{X}$.

\section{Problem Statement}

We consider discrete time Markovian switching systems with dynamics of the form

$$
x_{t+1}=f\left(x_{t}, u_{t}, w_{t+1}\right),
$$

where $x_{t} \in \mathbb{R}^{n_{x}}, u_{t} \in \mathbb{R}^{n_{u}}$ are the state and control action at time $t$, respectively, and $w_{t+1}: \Omega \rightarrow W$ is a random variable drawn from a discrete-time, time-homogeneous Markov chain $\mathbf{w}:=\left(w_{t}\right)_{t \in \mathbb{N}}$ defined on a probability space $(\Omega, \mathcal{F}, \mathbb{P})$ and taking values on $W:=\mathbb{N}_{[1, M]}$. We refer to $w_{t}$ as the mode of the Markov chain at time $t$. The transition kernel governing the Markov chain is denoted by $P=\left(P_{i j}\right)_{i, j \in W}$, where $P_{i j}=\mathbb{P}\left[w_{t}=j \mid w_{t-1}=i\right]$. As such, the probability space can be constructed by taking the sample space $\Omega=$ $W^{\infty}, \mathcal{F}=2^{\Omega}$ and for any $\left(w_{t}\right)_{t \in \mathbb{N}} \in \Omega, \mathbb{P}\left[\left(w_{t}\right)_{t \in \mathbb{N}}\right]=$ $p_{0} \prod_{t=0}^{\infty} P_{w_{t} w_{t+1}}$, where $p_{0} \in \Delta_{M}$ is the initial distribution [22]. For simplicity, we will assume that the initial mode is known to be $w$, so $p_{0}=\left(1_{i=w}\right)_{i \in W}$. We assume that the state $x_{t}$ and mode $w_{t}$ are observable at time $t$.

For a given state-mode pair $(x, w) \in \mathbb{R}^{n_{x}} \times W$, we assume that the control action $u$ is constrained to lie within the set $\mathcal{U}(x, w)$, defined through the chance constraints

$$
\begin{aligned}
& \mathcal{U}(x, w):=\left\{u \in \mathbb{R}^{n_{u}}:\right. \\
& \left.\quad \mathbb{P}\left[g_{i}\left(x, u, w, w^{\prime}\right)>0 \mid x, w\right]<\alpha_{i}, \forall i \in \mathbb{N}_{\left[1, n_{g}\right]}\right\},
\end{aligned}
$$

where $w^{\prime}: \Omega \rightarrow W$ is a random variable distributed according to $P_{w}$ : and $g_{i}: \mathbb{R}^{n_{x}} \times \mathbb{R}^{n_{u}} \times W \times W \rightarrow \mathbb{R}$ are constraint functions with corresponding constraint violation rates $\alpha_{i}$. By appropriate choices of $\alpha_{i}$ and $g_{i}$, constraint (2) can be used to encode robust constraints $\left(\alpha_{i}=0\right)$ or chance constraints $\left(0<\alpha_{i}<1\right)$ on the state, the control action or both. Note that the formulation (2) covers chance constraints on the successor state $f\left(x, u, w^{\prime}\right)$ under input $u$, conditioned on the values $x$ and $w$.

Ideally, our goal is to synthesize - by means of a stochastic MPC scheme - a stabilizing control law $\kappa$ : $\mathbb{R}^{n_{x}} \times W \rightarrow \mathbb{R}^{n_{u}}$, such that for the closed loop system $x_{t+1}=f\left(x_{t}, \kappa\left(x_{t}, w_{t}\right), w_{t+1}\right)$, it holds almost surely (a.s.) that $\kappa\left(x_{t}, w_{t}\right) \in \mathcal{U}\left(x_{t}, w_{t}\right)$, for all $t \in \mathbb{N}$. Consider a sequence of $N$ control laws $\pi=\left(\kappa_{k}\right)_{k=0}^{N-1}$, referred to as a policy of length $N$. Given a stage cost $\ell: \mathbb{R}^{n_{x}} \times \mathbb{R}^{n_{u}} \times W \rightarrow$ $\mathbb{R}_{+}$, and a terminal cost function $\bar{V}_{\mathrm{f}}: \mathbb{R}^{n_{u}} \times W \rightarrow \overline{\mathbb{R}}_{+}$: $(x, w) \mapsto V_{\mathrm{f}}(x, w)+\delta_{\mathcal{X}_{\mathrm{f}}}(x)$, we can assign to each such policy $\pi$, a cost

$$
V_{N}^{\pi}(x, w):=\mathbb{E}\left[\sum_{k=0}^{N-1} \ell\left(x_{k}, u_{k}, w_{k}\right)+\bar{V}_{\mathrm{f}}\left(x_{N}, w_{N}\right)\right],
$$

where $x_{k+1}=f\left(x_{k}, u_{k}, w_{k+1}\right)$ and $u_{k}=\kappa_{k}\left(x_{k}, w_{k}\right)$ for all $k \in \mathbb{N}_{[0, N-1]}$. This defines the following optimal control problem (OCP).
Definition II.1 (Stochastic OCP). For a given state-mode pair $(x, w)$, the optimal cost of the stochastic OCP is

$$
V_{N}(x, w)=\min _{\pi} V_{N}^{\pi}(x, w)
$$

subject to

$$
\begin{aligned}
x_{0} & =x, w_{0}=w, \pi=\left(\kappa_{k}\right)_{k=0}^{N-1}, \\
x_{k+1} & =f\left(x_{k}, \kappa_{k}\left(x_{k}, w_{k}\right), w_{k+1}\right), \\
\kappa_{k}\left(x_{k}, w_{k}\right) & \in \mathcal{U}\left(x_{k}, w_{k}\right), \forall k \in \mathbb{N}_{[0, N-1]} .
\end{aligned}
$$

We denote by $\Pi_{N}(x, w)$ the corresponding set of minimizers.

Let $\left(\kappa_{k}^{\star}(x, w)\right)_{k=0}^{N-1} \in \Pi_{N}(x, w)$, so that the stochastic MPC control law is given by $\kappa_{N}(x, w)=\kappa_{0}^{\star}(x, w)$. Sufficient conditions on the terminal cost $\bar{V}_{\mathrm{f}}$ and its effective domain $\operatorname{dom} \bar{V}_{\mathrm{f}}$ for mean-square stability of the closed-loop, have been studied for a similar problem set-up in [4], among others.

Both designing and computing such a stochastic MPC law, however, requires knowledge of the probability distribution governing the state dynamics (1), or equivalently, of the transition kernel $P$. In reality, the transition probabilities of the Markov chain are typically not known but rather estimated from a finitely-sized sequence $w_{[0, t]}$ of observed values. Therefore, they are subject to some level of misestimation, which we refer to as ambiguity. Throughout the paper, we will make the following assumption to explicitly take this ambiguity into account.

Assumption II.2 (Learning system). Given a sequence $w_{[0, t]}$ sampled from the Markov chain $\mathbf{w}$, we can compute (i) a statistic $s_{t}: W^{t+1} \rightarrow \mathcal{S} \subseteq \mathbb{R}^{n_{s}}$, accompanied by a vector of confidence parameters $\beta_{t} \in \mathcal{I}:=[0,1]^{n_{\beta}}$, which admit recursive update rules $s_{t+1}=\mathcal{L}\left(s_{t}, w_{t}, w_{t+1}\right)$ and $\beta_{t+1}=C\left(\beta_{t}\right), t \in \mathbb{N}$; and (ii) an ambiguity set $\mathcal{A}: \mathcal{S} \times W \times[0,1] \rightrightarrows \Delta_{M}:(s, w, \beta) \mapsto \mathcal{A}_{\beta}(s, w)$, mapping $s_{t}, w_{t}$ and an element $\beta_{t}^{i}$ to a convex subset of the $M$ dimensional probability simplex such that for all $t \in \mathbb{N}$,

$$
\mathbb{P}\left[P_{w_{t}}: \in \mathcal{A}_{\beta_{t}^{i}}\left(s_{t}, w_{t}\right)\right] \geq 1-\beta_{t}^{i} .
$$

We will refer to $s_{t}$ and $\beta_{t}$ as the state of the learner and the confidence vector at time $t$, respectively.

Remark II.3 (confidence levels). Two points of clarification are in order. First, the choice of allowing a vector of confidence levels rather than a single value is motivated by the fact that one would typically wish to assign different confidence levels to different ambiguity sets corresponding to the individual constraints and the cost function of the data-driven OCP (defined below in Definition III.4).

Second, the confidence levels are completely exogenous to the system dynamics and can in principle be chosen to be any time-varying sequence satisfying the conditions of Proposition III.1 and Lemma IV.5 to ensure constraint satisfaction and stability, respectively. The requirement that $\left(\beta_{t}\right)_{t \in \mathbb{N}}$ can be written as the trajectory of a time-invariant dynamical system is only mildly restrictive (see Example II.4) and posed mainly for notational convenience when analyzing the theoretical properties of the proposed control scheme. 
The idea of the learner state is very similar in spirit to the concept of a belief state, which is commonly used in control of partially observed Markov decision processes [21]. It can be regarded as a state that stores all the information required to build a set of possible conditional distributions over the next state. To fix ideas, consider the following example of a learning system satisfying the requirements of Assumption II.2.

Example II.4 (Transition counts and $\ell_{1}$-ambiguity). A natural choice for the learner state is to take $s_{t}=\operatorname{vec}\left(m_{t}\right)$, where $\mathbb{N}^{M \times M} \ni m_{t}=\left(m_{t, i j}\right)_{i, j \in M}$ contains the mode transition counts at time $t$. That is, $m_{t, i j}=\mid\left\{\tau \in \mathbb{N}_{[1, t]} \mid w_{\tau-1}=\right.$ $\left.i, w_{\tau}=j\right\} \mid$, for all $i, j \in M$. In this case, since

$$
m_{t+1, i j}= \begin{cases}m_{t, i j}+1 & \text { if } w_{t}=i, w_{t+1}=j \\ m_{t, i j} & \text { otherwise, }\end{cases}
$$

for all $i, j \in M$, we can indeed write $s_{t+1}=\mathcal{L}\left(s_{t}, w_{t}, w_{t+1}\right)$. Furthermore, following [23], we can uniquely obtain ambiguity sets parametrized as

$$
\mathcal{A}_{\beta_{t}^{i}}^{\ell_{1}}\left(s_{t}, w_{t}\right):=\left\{p \in \Delta_{M}:\left\|p-\hat{p}_{w_{t}}\right\|_{1} \leq r_{w_{t}}\left(s_{t}, \beta_{t, i}\right)\right\},
$$

for $i \in \mathbb{N}_{\left[1, n_{\beta}\right]}$, where $\hat{p}_{w_{t}}$ is the empirical estimate of the $w_{t}$ 'th row of the transition kernel (initialized to the uniform distribution if no transitions originating in mode $w_{t}$ have been observed) and the radii $r_{w_{t}}\left(s_{t}, \beta_{t, i}\right)$ are chosen to satisfy (5) by means of basic concentration inequalities. Both of these quantities depend solely on the learner state $s_{t}$, the confidence level $\beta_{t}$ and the current mode $w_{t}$.

The confidence levels would typically be chosen to be a monotone decreasing sequence. A particular family of sequences satisfying Assumption IV.4, is $\beta_{t}=b(1+t)^{-q}$, with some parameters $b \in[0,1], q>1, t \in \mathbb{N}$. It is easy to show that this sequence can indeed be written recursively as

$$
\beta_{t+1}=\frac{b \beta_{t}}{\left(\beta_{t}^{1 / q}+b^{1 / q}\right)^{q}}, \beta_{0}=b .
$$

Equipped with such a generic learning system, our aim is to find a data-driven approximation to the stochastic OCP defined by (4), which asymptotically attains the optimal cost, while preserving stability and constraint satisfaction during closed-loop operation.

\section{DATA-DRIVEN MODEL PREDICTIVE CONTROL}

Given a learning system satisfying Assumption II.2, we define the augmented state $z_{t}=\left(x_{t}, s_{t}, \beta_{t}\right) \in \mathcal{Z}:=\mathbb{R}^{n_{x}} \times$ $\mathcal{S} \times \mathcal{I}$, which evolves over time according to the dynamics

$$
z_{t+1}=\tilde{f}\left(z_{t}, u_{t}, w_{t}, w_{t+1}\right):=\left[\begin{array}{c}
f\left(x_{t}, u_{t}, w_{t+1}\right) \\
\mathcal{L}\left(s_{t}, w_{t}, w_{t+1}\right) \\
C\left(\beta_{t}\right)
\end{array}\right] .
$$

Consequently, our scheme will result in a feedback law $\kappa: \mathcal{Z} \times W \rightarrow \mathbb{R}^{n_{u}}$. To this end, we will formulate a distributionally robust counterpart to the stochastic OCP (4), in which the expectation operator in the cost and the conditional probabilities in the constraint will be replaced by operators that account for ambiguity in the involved distributions.

\section{A. Ambiguity and risk}

In order to reformulate the cost function (3), we first introduce an ambiguous conditional expectation operator, leading to a formulation akin to the Markovian risk measures utilized in [8], [24]. Consider a function $\xi: \mathcal{Z} \times W^{2} \rightarrow \overline{\mathbb{R}}$, defining a stochastic process $\left(\xi_{t}\right)_{t \in \mathbb{N}}=\left(\xi\left(z_{t}, w_{t}, w_{t+1}\right)\right)_{t \in \mathbb{N}}$ on $(\Omega, \mathcal{F}, \mathbb{P})$, and suppose that the augmented state $\left(z_{t}, w_{t}\right)=$ $(z, w)=(x, s, \beta, w)$ is given. For simplicity, let us assume for the moment that $\beta \in[0,1]$ is scalar; the following definition can be repeated for each component in the general case. The ambiguous conditional expectation of $\xi$, given $z, w$ is defined

$$
\begin{aligned}
\rho_{s, w}^{\beta}\left[\xi\left(z, w, w^{\prime}\right)\right] & :=\max _{p \in \mathcal{A}_{\beta}(s, w)} \mathbb{E}_{p}\left[\xi\left(z, w, w^{\prime}\right) \mid z, w\right] \\
& =\max _{p \in \mathcal{A}_{\beta}(s, w)} \sum_{w^{\prime} \in W} p^{w^{\prime}} \xi\left(z, w, w^{\prime}\right) .
\end{aligned}
$$

Trivially, it holds that if $P_{w:} \in \mathcal{A}_{\beta}(s, w)$, then

$$
\begin{aligned}
\rho_{s, w}^{\beta}\left[\xi\left(z, w, w^{\prime}\right)\right] & \geq \mathbb{E}_{P_{w:}}\left[\xi\left(z, w, w^{\prime}\right) \mid z, w\right] \\
& =\sum_{w^{\prime} \in W} P_{w w^{\prime}} \xi\left(z, w, w^{\prime}\right) .
\end{aligned}
$$

Note that the function $\rho_{s, w}^{\beta}$ defines a coherent risk measure [25, Sec. 6.3]. We say that $\rho_{s, w}^{\beta}$ is the risk measure induced by the ambiguity set $\mathcal{A}_{\beta}(s, w)$.

A similar construction can be carried out for the chance constraints (2). In their standard form, chance constraints lead to nonconvex, nonsmooth constraints. For this reason, they are commonly replaced using risk measures [26]. Particularly, the (conditional) average value-at-risk (at level $\hat{\alpha} \in(0,1]$ and with reference distribution $p \in \Delta_{M}$ ) of $\xi$ is the coherent risk measure

$$
\begin{aligned}
& \mathrm{AV} @ \mathrm{R}_{\hat{\alpha}}^{p}\left[\xi\left(z, w, w^{\prime}\right) \mid z, w\right] \\
& = \begin{cases}\min _{t \in \mathbb{R}} t+1 / \hat{\alpha} \mathbb{E}_{p}\left[\xi\left(z, w, w^{\prime}\right)-t \mid z, w\right]_{+}, & \hat{\alpha} \neq 0 \\
\max _{w^{\prime} \in W}\left[\xi\left(z, w, w^{\prime}\right)\right], & \hat{\alpha}=0 .\end{cases}
\end{aligned}
$$

It can be shown (e.g., [25, sec. 6.2.4]) that if $p=P_{w \text { : }}$, then the following implication holds tightly

$$
\begin{aligned}
& \mathrm{AV} @ \mathrm{R}_{\hat{\alpha}}^{p}\left[\xi\left(z, w, w^{\prime}\right) \mid z, w\right] \leq 0 \\
& \Rightarrow \mathbb{P}\left[\xi\left(z, w, w^{\prime}\right) \leq 0 \mid z, w\right] \geq 1-\hat{\alpha} .
\end{aligned}
$$

By exploiting the dual risk representation [25, Thm 6.5], the left-hand inequality in (9) can be formulated in terms of only linear constraints [9]. As such, it can be used as a tractable surrogate for the original chance constraints, given perfect probabilistic information. Accounting also for the ambiguity in the knowledge of $P_{w}$ : through $\mathcal{A}_{\beta}(s, w)$, we define

$$
\bar{\rho}_{s, w}^{\beta, \hat{\alpha}}\left[\xi\left(z, w, w^{\prime}\right)\right]:=\max _{p \in \mathcal{A}_{\beta}(s, w)} \operatorname{AV} @ \mathrm{R}_{\hat{\alpha}}^{p}\left[\xi\left(z, w, w^{\prime}\right) \mid z, w\right] \leq 0 .
$$

The function $\bar{\rho}_{s, w}^{\beta, \hat{\alpha}}$ in turn defines a coherent risk measure.

We now present a condition on the choice of $\hat{\alpha}$ under which a constraint of the form (10) can be used as a tractable and safe approximation of a chance constraint when there is ambiguity in the probability distribution.

Proposition III.1. Let $\beta, \alpha \in[0,1]$ be given values. Consider the random variable $s: \Omega \rightarrow \mathcal{S}$, denoting an (a priori 
unknown) learner state satisfying Assumption II.2, i.e., $\mathbb{P}\left[P_{w:} \in \mathcal{A}_{\beta}(s, w)\right] \geq 1-\beta$. If the parameter $\hat{\alpha}$ is chosen to satisfy $0 \leq \hat{\alpha} \leq \frac{\alpha-\beta}{1-\beta} \leq 1$, then, for an arbitrary function $g: \mathcal{Z} \times W^{2} \rightarrow \overline{\mathbb{R}}$, the following implication holds:

$$
\begin{array}{r}
\bar{\rho}_{s, w}^{\beta, \hat{\alpha}}\left[g\left(z, w, w^{\prime}\right)\right] \leq 0, \text { a.s. } \\
\Rightarrow \mathbb{P}\left[g\left(z, w, w^{\prime}\right) \leq 0 \mid x, w\right]>1-\alpha .
\end{array}
$$

Proof. If $\bar{\rho}_{s, w}^{\beta, \hat{\alpha}}\left[g\left(z, w, w^{\prime}\right)\right] \leq 0$, a.s., then by (9)-(10),

$$
\mathbb{P}\left[g\left(z, w, w^{\prime}\right) \leq 0 \mid x, w, P_{w:} \in \mathcal{A}_{\beta}(s, w)\right] \geq 1-\hat{\alpha}, \text { a.s. }
$$

Therefore,

$$
\begin{aligned}
& \mathbb{P}\left[g\left(z, w, w^{\prime}\right) \leq 0 \mid x, w\right] \\
& =\mathbb{P}\left[g\left(z, w, w^{\prime}\right) \leq 0 \mid x, w, P_{w:} \in \mathcal{A}_{\beta}(s, w)\right] \\
& \quad \quad \mathbb{P}\left[P_{w:} \in \mathcal{A}_{\beta}(s, w)\right] \\
& \geq(1-\hat{\alpha})(1-\beta) .
\end{aligned}
$$

Requiring that $(1-\hat{\alpha})(1-\beta) \geq(1-\alpha)$ then immediately yields the sought condition.

Remark III.2. Notice that Proposition III.1 provides an $a$ priori guarantee, in that the learner state is assumed to be random. For a given learner state $s$ and mode $w$, the ambiguity set $\mathcal{A}_{\beta}(s, w)$ is fixed and therefore, the outcome of the event $\left\{P_{w:} \in \mathcal{A}_{\beta}(s, w)\right\}$ is determined. This manifests itself in the control design through the above condition which implies that $\hat{\alpha} \leq \alpha$, and thus tightens the chance constraints for a fixed $s$. Hence, the possibility that for this particular $s$, the ambiguity set may not include the conditional distribution, is accounted for. This effect can be mitigated by decreasing $\beta$, at the cost of a larger ambiguity set. A more detailed study of this trade-off for different types of ambiguity sets is left for future work.

\section{B. Risk-averse optimal control}

We are now ready to describe the distributionally robust counterpart to the OCP (4), which, when solved in receding horizon fashion, yields the proposed data-driven MPC scheme.

For a given augmented state and mode $(z, w)=$ $(x, s, \beta, w) \in \mathcal{Z} \times W$, we use (10) to define the distributionally robust set of feasible inputs $\hat{\mathcal{U}}(z, w)$ in correspondence to (2). Let $d: \mathbb{N}_{\left[1, n_{g}\right]} \rightarrow \mathbb{N}_{\left[1, n_{\beta}\right]}$ be an arbitrary injective mapping, assigning the entry $\beta^{d(i)}$ of the confidence vector $\beta$ to the $i$ 'th constraint, so that

$$
\hat{\mathcal{U}}(z, w)=\left\{u \mid \bar{\rho}_{s, w}^{\beta^{d(i)}, \hat{\alpha}_{i}}\left[g_{i}\left(x, u, w, w^{\prime}\right)\right] \leq 0, \forall i \in \mathbb{N}_{\left[1, n_{g}\right]}\right\} .
$$

Remark III.3. In light of Proposition III.1, $\hat{\alpha}_{i}=\frac{\alpha_{i}-\beta^{d(i)}}{1-\beta^{d(i)}}$ yields the least conservative choice. This choice is valid as long as it is ensured that $\beta^{d(i)} \leq \alpha_{i}$.

Similarly, consider some fixed index $v \in \mathbb{N}_{\left[1, n_{g}\right]}$, indicating the entry in the confidence vector associated to the cost. Using (7), we can then express the distributionally robust cost of a policy $\pi=\left(\kappa_{k}\right)_{k=0}^{N-1}$ as

$$
\begin{aligned}
\hat{V}_{N}^{\pi}(z, w):= & \ell\left(x_{0}, u_{0}, w_{0}\right)+\rho_{s_{0}, w_{0}}^{\beta_{0}^{v}}\left[\ell\left(x_{1}, u_{1}, w_{1}\right)\right. \\
+\rho_{s_{1}, w_{1}}^{\beta_{1}^{v}} & {\left[\cdots+\rho_{s_{N-2}, w_{N-2}}^{\beta_{N-2}^{v}}\left[\ell\left(x_{N-1}, u_{N-1}, w_{N-1}\right)\right.\right.} \\
& \left.\left.\left.+\rho_{s_{N}, w_{N}}^{\beta_{N}^{v}}\left[\hat{V}_{\mathrm{f}}\left(x_{N}, s_{N}, \beta_{N}, w_{N}\right)\right]\right] \ldots\right]\right]
\end{aligned}
$$

where $\left(z_{0}, w_{0}\right)=(z, w), z_{k+1}=\tilde{f}\left(z_{k}, u_{k}, w_{k}, w_{k+1}\right)$ and $u_{k}=\kappa_{k}\left(z_{k}, w_{k}\right)$, for all $k \in \mathbb{N}_{[0, N-1]}$. In Section IV, conditions on the terminal cost $\hat{V}_{\mathrm{f}}: \mathcal{Z} \times W \rightarrow \overline{\mathbb{R}}_{+}$: $(x, s, \beta, w) \mapsto V_{\mathrm{f}}(x, w)+\delta_{\hat{\mathcal{X}}_{\mathrm{f}}}(x, s, \beta)$ and its domain are provided in order to guarantee recursive feasibility and stability of the MPC scheme defined by the following OCP.

Definition III.4 (DR-OCP). Given an augmented state-mode pair $(z, w) \in \mathcal{Z} \times W$, the optimal cost of the distributionally robust optimal control problem $(D R-O C P)$ is

$$
\hat{V}_{N}(z, w)=\min _{\pi} \hat{V}_{N}^{\pi}(z, w)
$$

subject to

$$
\begin{aligned}
\left(x_{0}, s_{0}, \beta_{0}, w_{0}\right) & =(z, w), \pi=\left(\kappa_{k}\right)_{k=0}^{N-1}, \\
z_{k+1} & =\tilde{f}\left(z_{k}, \kappa_{k}\left(z_{k}, w_{k}\right), w_{k}, w_{k+1}\right), \\
\kappa_{k}\left(z_{k}, w_{k}\right) & \in \hat{\mathcal{U}}\left(z_{k}, w_{k}\right), \forall w_{[0, k]} \in W^{k},
\end{aligned}
$$

for all $k \in \mathbb{N}_{[0, N-1]}$. We denote by $\hat{\Pi}_{N}(z, w)$ the corresponding set of minimizers.

We thus define the data-driven MPC law analogously to the stochastic case as

$$
\hat{\kappa}_{N}(z, w)=\hat{\kappa}_{0}^{\star}(z, w),
$$

where $\left(\hat{\kappa}_{k}^{\star}(z, w)\right)_{k=0}^{N-1} \in \hat{\Pi}_{N}(z, w)$. At every time $t$, the data-driven MPC scheme thus consists of (i) computing a control action $u_{t}=\hat{\kappa}_{N}\left(z_{t}, w_{t}\right)$ and applying it to the system (1); (ii) observing the outcome of $w_{t+1} \in W$ and the corresponding next state $x_{t+1}=f\left(x_{t}, u_{t}, w_{t+1}\right)$; and (iii) updating the learner state $s_{t+1}=\mathcal{L}\left(s_{t}, w_{t}, w_{t+1}\right)$ and the confidence levels $\beta_{t+1}=C\left(\beta_{t}\right)$, gradually decreasing the size of the ambiguity sets.

Remark III.5 (Scenario tree representations). Note that since $W$ is a finite set, the possible realizations of $w_{[0, N]}$, given $\left(z_{0}, w_{0}\right)$ and by extension the corresponding predicted states and controls can be represented on a scenario tree, which is a directed graph with a single root node, corresponding to $w_{0}$. Each node is connected to a number of child nodes, representing the possible realizations of the stochastic process at the next time step [27]. It therefore suffices to optimize over a finite number (equal to the number of modes) of control actions and instead of full control laws.

When represented in this manner, it is clear that (13) falls within the class of risk-averse, risk-constrained optimal control problems, described in [9]. In particular, the constraints (13d) at stage $k$ can be represented in the framework of [9] as nested risk constraints which is a composition of a set of conditional risk mappings, in this case consisting of 
$k-1$ max operators and a conditional risk mapping based on (10) at stage $k$. This is in line with the observations of [28, Sec. 7.1]. Consequently, if the risk measures employed in the definition of the DR-OCP (13) belong to the broad family of conic risk measures, then the reformulations in [9] can be applied to cast (13) as a conic optimization problem which can be effectively solved with available solvers. This is the case for many commonly used coherent risk measures, including the risk measure induced by the $\ell_{1}$-ambiguity set discussed in Example II.4.

\section{THEORETICAL ANALYSIS}

\section{A. Dynamic programming}

To facilitate theoretical analysis of the proposed MPC scheme, we represent (13) as a dynamic programming recursion, similarly to [8]. We define the Bellman operator $\mathbf{T}$ as

$\mathbf{T}(\hat{V})(z, w):=\min _{u \in \hat{\mathcal{U}}(z, w)} \ell(x, u, w)+\rho_{s, w}^{\beta^{c}}\left[\hat{V}\left(\tilde{f}\left(z, u, w, w^{\prime}\right), w^{\prime}\right)\right]$,

where $(z, w)=(x, s, \beta, w) \in \mathcal{Z} \times W$, are fixed quantities and $w^{\prime}$ is a random variable with distribution $P_{w:}$. Let $\mathbf{S}(\hat{V})(z, w)$ denote the corresponding set of minimizers. The optimal cost $\hat{V}_{N}$ of (13) is thus obtained through the iteration,

$$
\hat{V}_{k}=\mathbf{T} \hat{V}_{k-1}, \hat{V}_{0}=\hat{V}_{\mathrm{f}}, k \in \mathbb{N}_{[1, N]}
$$

Similarly, $\mathcal{X}_{k}(w):=\operatorname{dom} \hat{V}_{k}(\cdot, w)$ is given recursively by

$$
\mathcal{X}_{k}(w)=\left\{z \mid \exists u \in \hat{\mathcal{U}}(z, w): \begin{array}{c}
\tilde{f}\left(z, u, w, w^{\prime}\right) \in \mathcal{X}_{k-1}\left(w^{\prime}\right) \\
\forall w^{\prime} \in W
\end{array}\right\} .
$$

Now consider the stochastic closed-loop system

$$
z_{t+1}=\tilde{f}^{\hat{\kappa}_{N}}\left(z_{t}, w_{t}, w_{t+1}\right):=\tilde{f}\left(z_{t}, \hat{\kappa}_{N}\left(z_{t}, w_{t}\right), w_{t}, w_{t+1}\right) \text {, }
$$

where $\hat{\kappa}_{N}\left(z_{t}, w_{t}\right) \in \mathbf{S}\left(\hat{V}_{N-1}\right)\left(z_{t}, w_{t}\right)$ is an optimal control law obtained by solving the data-driven DR-OCP of horizon $N$ in receding horizon. In the remainder of this section, we will show that Assumption II.2 allows us to establish several desired properties of (16), despite only having incomplete knowledge of the transition probabilities.

\section{B. Constraint satisfaction and recursive feasibility}

In order to show existence of $\hat{\kappa}_{N} \in \mathbf{S} \hat{V}_{N-1}$ at every time step, Proposition IV.2 will require that $\mathcal{X}_{\mathrm{f}}$ is a robust control invariant set. We define robust control invariance for the augmented control system under consideration as follows.

Definition IV.1 (Robust invariance). A set $\mathcal{R} \subseteq \mathcal{Z}$ is called a robust control invariant (RCI) set for the system (6) if for all $(x, w) \in \mathcal{R} \times W$, there exists a $u \in \hat{\mathcal{U}}(z, w)$ such that $\tilde{f}\left(z, u, w, w^{\prime}\right) \in \mathcal{R}, \forall w^{\prime} \in W$. Similarly, we say that $\mathcal{R}$ is a robust positive invariant (RPI) set for the closed-loop system (16) iffor all $(z, w) \in \mathcal{R} \times W, \tilde{f}^{\hat{\kappa}_{N}}\left(z, w, w^{\prime}\right) \in \mathcal{R}, \forall w^{\prime} \in W$.

Proposition IV.2 (Recursive feasibility). Suppose that $\mathcal{X}_{\mathrm{f}}$ is a RCI set for (6). Then, (13) is recursively feasible, that is, feasibility of DR-OCP (13) for some state-mode pair $(z, w) \in$ $\mathcal{Z} \times W$, implies feasibility for $\left(\tilde{f}^{\hat{\kappa}_{N}}\left(z, w, w^{\prime}\right), w^{\prime}\right)$, for all $w^{\prime} \in W, N \in \mathbb{N}_{>0}$.
Proof. We first show that if $\mathcal{X}_{\mathrm{f}}$ is RCI, then so is $\mathcal{X}_{N}(w)$, where $w$ is the given initial mode. This is done by induction on the horizon $N$ of the OCP.

Base case $(N=0) . \mathcal{X}_{0}(w)=\mathcal{X}_{\mathrm{f}}$ for all $w \in W$ and $\mathcal{X}_{\mathrm{f}}$ is RCI.

Induction step $(N \Rightarrow N+1)$. Suppose that for some $N \in$ $\mathbb{N}, \mathcal{X}_{N}(w)$ is RCI for (6). Then, by definition of $\mathcal{X}_{N+1}$, for each $z \in \mathcal{X}_{N+1}(w)$, there exists a nonempty set $\hat{\mathcal{U}}_{N}^{\star}(z, w) \subseteq$ $\hat{\mathcal{U}}(z, w)$ such that for every $u \in \hat{\mathcal{U}}_{N}^{\star}(z, w)$ and for all $w^{\prime} \in$ $W, z^{\prime} \in \mathcal{X}_{N}\left(w^{\prime}\right)$, where $z^{\prime}=\tilde{f}\left(z, u, w, w^{\prime}\right)$. Furthermore, the induction hypothesis $\left(\mathcal{X}_{N}\right.$ is RCI), implies that there also exists a $u^{\prime} \in \hat{\mathcal{U}}\left(z^{\prime}, w^{\prime}\right)$ such that $\tilde{f}\left(z^{\prime}, u^{\prime}, w^{\prime}, w^{\prime \prime}\right) \in$ $\mathcal{X}_{N}\left(w^{\prime \prime}\right), \forall w^{\prime \prime} \in W$. Therefore, $z^{\prime}$ satisfies the conditions defining $\mathcal{X}_{N+1}$. In other words, $\mathcal{X}_{N+1}$ is RCI.

The claim follows from the fact that for any $N>0, w \in W$ and $z \in \mathcal{X}_{N}(w), \hat{\kappa}_{N}(z, w) \in \mathbf{S} \hat{V}_{N-1}(z, w) \subseteq \hat{\mathcal{U}}_{N-1}^{\star}(z, w)$, as any other choice of $u$ would yield infinite cost in the definition of the Bellman operator.

Corollary IV.3 (Chance constraint satisfaction). If Proposition IV.2 holds, then by Proposition III.1, the stochastic process $\left(z_{t}, w_{t}\right)_{t \in \mathbb{N}}=\left(x_{t}, s_{t}, \beta_{t}, w_{t}\right)_{t \in \mathbb{N}}$ following (16) satisfies the chance constraints

$$
\mathbb{P}\left[g_{i}\left(x_{t}, \hat{\kappa}_{N}\left(z_{t}, w_{t}\right), w_{t}, w_{t+1}\right)>0 \mid x_{t}, w_{t}\right]<\alpha_{i},
$$

a.s., for all $i \in \mathbb{N}_{\left[1, n_{g}\right]}, t \in \mathbb{N}$.

We conclude this section by emphasizing that although the MPC scheme guarantees closed-loop constraint satisfaction, it does so while being less conservative than a fully robust approach, which neglects statistical information. Indeed, we recover a robust approach by taking $\mathcal{A}_{\beta}(s, w)=\Delta_{M}$ for all $s, w, \beta$. It is apparent from (10) and (11), that for all other choices of the ambiguity set, the set of feasible control actions will be larger (in the sense of set inclusion).

\section{Stability}

In this section, we will provide sufficient conditions on the control set-up under which the origin is mean-square stable (MSS) for (16), i.e., $\lim _{t \rightarrow \infty} \mathbb{E}\left[\left\|x_{t}\right\|\right]=0$ for all $x_{0}$ in some specified compact set containing the origin. To do this, we will make the following assumption.

Assumption IV.4. The confidence dynamics $\beta_{t+1}=C\left(\beta_{t}\right)$ is chosen such that $\sum_{t=0}^{\infty} \beta_{t}^{i}<\infty, \forall i \in \mathbb{N}_{\left[1, n_{\beta}\right]}$.

This assumption guarantees that the probability (5) of capturing the true conditional distribution in the constructed ambiguity set increases sufficiently fast. This presents a tradeoff with performance considerations. Statistical divergencebased ambiguity sets (e.g., Example II.4) typically have a radius $r_{t} \sim \sqrt{-t^{-1} \ln \left(\beta_{t}\right)}$. Requiring that $r_{t} \rightarrow 0$ as $t \rightarrow \infty$, yields a lower bound on how fast $\beta_{t}$ may decrease.

Our main stability result, stated in Theorem IV.6, hinges in large on the following lemma, which relates risk-square stability [8, Lem. 5] of the origin for the autonomous system (16) to stability in the mean-square sense, based on the statistical properties of the ambiguity sets. 
Lemma IV.5 (Distributionally robust MSS condition). Suppose that Assumption IV.4 holds and that there exists a nonnegative, proper function $V: \mathbb{R}^{n_{x}} \times \mathcal{S} \times \mathcal{I} \times W \rightarrow \overline{\mathbb{R}}_{+}$, such that,

(i) $\operatorname{dom} V \subseteq \mathcal{X} \times \mathcal{S} \times \mathcal{I} \times W=\mathcal{Z} \times W$, where $\mathcal{Z}$ is RPI for system (16) and $\mathcal{X} \subset \mathbb{R}^{n_{x}}$ is a compact set containing the origin;

(ii) $\rho_{s, w}^{\beta^{v}}\left[V\left(\tilde{f}^{\hat{\kappa}_{N}}\left(z, w, w^{\prime}\right), w^{\prime}\right)\right]-V(z, w) \leq-c\|x\|^{2}$, for some $c>0$, for all $(z, w) \in \operatorname{dom} V$.

Then $\mathbb{E}\left[\sum_{t=0}^{k}\left\|x_{t}\right\|^{2}\right]$ is uniformly bounded in $k$ for all $z_{0} \in$ $\operatorname{dom} V\left(\cdot, w_{0}\right)$, where $\left(z_{t}, w_{t}\right)_{t \in \mathbb{N}}=\left(x_{t}, s_{t}, \beta_{t}, w_{t}\right)_{t \in \mathbb{N}}$ is the stochastic process governed by dynamics (16).

Proof. See Appendix.

Theorem IV.6 (MPC stability). Suppose that Assumption IV.4 is satisfied and the following statements hold.

(i) $\mathbf{T} \hat{V}_{\mathrm{f}} \leq \hat{V}_{\mathrm{f}}$;

(ii) $c\|x\|^{2} \leq \ell(x, u, w)$ for some $c>0$ for all $(x, s, \beta, w) \in$ $\operatorname{dom} \hat{V}_{N}, u \in \hat{\mathcal{U}}(x, w)$.

Then, the origin is MSS for the MPC-controlled system (16), over all RPI sets $\overline{\mathcal{Z}}:=\overline{\mathcal{X}} \times \mathcal{S} \times \mathcal{I}: \overline{\mathcal{Z}} \times W \subseteq \operatorname{dom} \hat{V}_{N}$, where $\overline{\mathcal{X}} \subseteq \mathbb{R}^{n_{x}}$ is a compact set containing the origin.

Proof. The proof is along the lines of that of [8, thm. 6] and shows that $\hat{V}_{N}$ satisfies the conditions of Lemma IV.5. Details are in the Appendix.

\section{CONCLUSIONS AND FUTURE WORK}

We have presented a data-driven, distributionally robust model predictive control strategy for Markovian switching systems with unknown transition probabilities subject to general chance constraints. Based on a data-driven ambiguity set, which includes the conditional probability vector over the next mode, given the current information, we derive a distributionally robust counterpart to a nominal stochastic MPC scheme. Using a dynamic programming formulation of the problem, we show that the distributionally robust MPC scheme can be used to provide a priori guarantees on closed-loop constraint satisfaction and mean-square stability of the true system, without requiring explicit knowledge of the transition probabilities.

In ongoing and future work, we aim to investigate the level of conservatism induced by different types of ambiguity sets, and extend the methodology to the case where the discrete mode cannot be observed directly. Furthermore, we plan to investigate parallelized solution methods for the discussed optimal control problems, which are still hindered by an exponential growth in the prediction horizon.

\section{REFERENCES}

[1] L. Hewing, K. P. Wabersich, M. Menner, and M. N. Zeilinger, "Learning-Based Model Predictive Control: Toward Safe Learning in Control," Annual Review of Control, Robotics, and Autonomous Systems, vol. 3, no. 1, 2020.

[2] O. L. d. V. Costa, M. D. Fragoso, and R. P. Marques, Discrete-time Markov jump linear systems. Probability and its applications, London: Springer, 2005.
[3] Y.-L. Chow and M. Pavone, "Stochastic optimal control with dynamic, time-consistent risk constraints," in 2013 American Control Conference, pp. 390-395, June 2013.

[4] P. Patrinos, P. Sopasakis, H. Sarimveis, and A. Bemporad, "Stochastic model predictive control for constrained discrete-time Markovian switching systems," Automatica, vol. 50, pp. 2504-2514, Oct. 2014.

[5] D. Bernardini and A. Bemporad, "Scenario-based model predictive control of stochastic constrained linear systems," in Proceedings of the 48 IEEE Conference on Decision and Control (CDC) Held Jointly with 2009 28th Chinese Control Conference, (Shanghai), pp. 6333-6338, IEEE, Dec. 2009.

[6] R. L. Beirigo, M. G. Todorov, and A. M. S. Barreto, "Online TD(A) for discrete-time Markov jump linear systems," in 2018 IEEE Conference on Decision and Control (CDC), pp. 2229-2234, Dec. 2018.

[7] S. He, M. Zhang, H. Fang, F. Liu, X. Luan, and Z. Ding, "Reinforcement learning and adaptive optimization of a class of Markov jump systems with completely unknown dynamic information," Neural Computing and Applications, Apr. 2019.

[8] P. Sopasakis, D. Herceg, A. Bemporad, and P. Patrinos, "Risk-averse model predictive control," Automatica, vol. 100, pp. 281-288, Feb. 2019.

[9] P. Sopasakis, M. Schuurmans, and P. Patrinos, "Risk-averse riskconstrained optimal control," in 2019 18th European Control Conference (ECC), pp. 375-380, June 2019.

[10] S. Singh, Y.-L. Chow, A. Majumdar, and M. Pavone, "A Framework for Time-Consistent, Risk-Sensitive Model Predictive Control: Theory and Algorithms," arXiv:1703.01029 [cs, math], Apr. 2018.

[11] H. Rahimian and S. Mehrotra, "Distributionally Robust Optimization: A Review," arXiv:1908.05659 [cs, math, stat], Aug. 2019.

[12] A. Ben-Tal, L. El Ghaoui, and A. S. Nemirovskiî, Robust Optimization. Princeton Series in Applied Mathematics, Princeton: Princeton University Press, 2009.

[13] I. Yang, "Wasserstein Distributionally Robust Stochastic Control: A Data-Driven Approach," arXiv:1812.09808 [cs, math], Dec. 2018.

[14] P. Mohajerin Esfahani and D. Kuhn, "Data-driven distributionally robust optimization using the Wasserstein metric: Performance guarantees and tractable reformulations," Mathematical Programming, vol. 171, pp. 115-166, Sept. 2018.

[15] B. Van Parys, D. Kuhn, P. Goulart, and M. Morari, "Distributionally Robust Control of Constrained Stochastic Systems," IEEE Transactions on Automatic Control, pp. 1-1, 2015.

[16] V. Borkar and R. Jain, "Risk-Constrained Markov Decision Processes," IEEE Transactions on Automatic Control, vol. 59, pp. 2574-2579, Sept. 2014.

[17] K. Uğurlu, "Robust optimal control using conditional risk mappings in infinite horizon," Journal of Computational and Applied Mathematics, vol. 344, pp. 275-287, Dec. 2018.

[18] H. Xu and S. Mannor, "Distributionally Robust Markov Decision Processes," in Advances in Neural Information Processing Systems 23 (J. D. Lafferty, C. K. I. Williams, J. Shawe-Taylor, R. S. Zemel, and A. Culotta, eds.), pp. 2505-2513, Curran Associates, Inc., 2010.

[19] A. Nilim and L. El Ghaoui, "Robust Control of Markov Decision Processes with Uncertain Transition Matrices," Operations Research, vol. 53, pp. 780-798, Oct. 2005.

[20] D. P. Bertsekas, Dynamic Programming and Optimal Control. Vol. 1: ... No. 3 in Athena Scientific Optimization and Computation Series, Belmont, Mass: Athena Scientific, 3. ed ed., 2005.

[21] V. Krishnamurthy, Partially Observed Markov Decision Processes: From Filtering to Controlled Sensing. Cambridge: Cambridge University Press, 2016.

[22] A. N. Shiriaev, Probability. No. 95 in Graduate texts in mathematics, New York: Springer, third edition ed., 2016.

[23] M. Schuurmans, P. Sopasakis, and P. Patrinos, "Safe Learning-Based Control of Stochastic Jump Linear Systems: A Distributionally Robust Approach," Mar. 2019.

[24] A. Ruszczyński, "Risk-averse dynamic programming for Markov decision processes," Mathematical Programming, vol. 125, pp. 235-261, Oct. 2010.

[25] A. Shapiro, D. Dentcheva, and A. Ruszczyński, Lectures on stochastic programming: modeling and theory. SIAM, 2009.

[26] A. Nemirovski, "On safe tractable approximations of chance constraints," European Journal of Operational Research, vol. 219, no. 3, pp. 707718, 2012.

[27] G. C. Pflug and A. Pichler, Multistage Stochastic Optimization. Springer 
Series in Operations Research and Financial Engineering, Cham: Springer International Publishing, 2014.

[28] B. Kouvaritakis and M. Cannon, Model Predictive Control. Advanced Textbooks in Control and Signal Processing, Cham: Springer International Publishing, 2016.

\section{APPENDIX}

\section{Proof of Lemma IV.5.}

Let $\left(z_{t}, w_{t}\right)_{t \in \mathbb{N}}=\left(x_{t}, s_{t}, \beta_{t}, w_{t}\right)_{t \in \mathbb{N}}$ denote the stochastic process satisfying dynamics (16), for some $\left(z_{0}, w_{0}\right) \in \operatorname{dom} V$. For ease of notation, let us define $V_{t}:=V\left(z_{t}, w_{t}\right), t \in \mathbb{N}$. Due to nonnegativity of $V$,

$$
\begin{aligned}
\mathbb{E}\left[\sum_{t=0}^{k-1} c\left\|x_{t}\right\|^{2}\right] & \leq \mathbb{E}\left[V_{k}+\sum_{t=0}^{k-1} c\left\|x_{t}\right\|^{2}\right] \\
& =\mathbb{E}\left[V_{k}-V_{0}+\sum_{t=0}^{k-1} c\left\|x_{t}\right\|^{2}\right]+V_{0},
\end{aligned}
$$

where the second equality follows from the fact that $V_{0}$ is deterministic. By linearity of the expectation, we can in turn write

$$
\begin{aligned}
\mathbb{E}\left[V_{k}-V_{0}+c \sum_{t=0}^{k-1}\left\|x_{t}\right\|^{2}\right] & =\mathbb{E}\left[\sum_{t=0}^{k-1} V_{t+1}-V_{t}+c\left\|x_{t}\right\|^{2}\right] \\
& =\sum_{t=0}^{k-1} \mathbb{E}\left[V_{t+1}-V_{t}+c\left\|x_{t}\right\|^{2}\right] .
\end{aligned}
$$

Therefore,

$$
\begin{aligned}
\mathbb{E}\left[c \sum_{t=0}^{k-1}\left\|x_{t}\right\|^{2}\right]-V_{0} & \leq \sum_{t=0}^{k-1} \mathbb{E}\left[V_{t+1}-V_{t}+c\left\|x_{t}\right\|^{2}\right] \\
& =\sum_{t=0}^{k-1} \mathbb{E}\left[V_{t+1}-V_{t}\right]+c \mathbb{E}\left[\left\|x_{t}\right\|^{2}\right] .
\end{aligned}
$$

Recall that $v \in \mathbb{N}_{\left[1, n_{\beta}\right]}$ specifies the coordinate of $\beta$ corresponding to the risk measures in the cost function (12). defining the event

$$
E_{t}:=\left\{\omega \in \Omega \mid P_{w_{t}(\omega)} \in \mathcal{A}_{\beta_{t}^{v}}\left(s_{t}(\omega), w_{t}(\omega)\right\},\right.
$$

and its complement $\neg E_{t}=\Omega \backslash E_{t}$, we can use the law of total expectation to write

$$
\begin{aligned}
\mathbb{E}\left[V_{t+1}-V_{t}\right]=\mathbb{E}\left[V_{t+1}-V_{t} \mid E_{t}\right] \mathbb{P}\left[E_{t}\right] & +\mathbb{E}\left[V_{t+1}-V_{t} \mid \neg E_{t}\right] \mathbb{P}\left[\neg E_{t}\right] .
\end{aligned}
$$

By condition (5), $\mathbb{P}\left[\neg E_{t}\right]<\beta_{t}^{v}$. From condition (i), it follows that $\left(z_{t}, w_{t}\right) \in \operatorname{dom} V, t \in \mathbb{N}_{[0, k]}$ and that there exists a $\bar{V} \geq 0$ such that $V(z, w) \leq \bar{V}$, for all $(z, w) \in \operatorname{dom} V$. Therefore, $\mathbb{E}\left[V_{t+1}-V_{t} \mid \neg \mathbb{E}_{t}\right] \leq \bar{V}$. Finally, by condition (ii), $\mathbb{E}\left[V_{t+1}-V_{t} \mid E_{t}\right] \leq \mathbb{E}\left[-c\left\|x_{t}\right\|^{2}\right]$. Thus,

$$
\mathbb{E}\left[V_{t+1}-V_{t}\right] \leq \mathbb{E}\left[-c\left\|x_{t}\right\|^{2} \mid E_{t}\right] \mathbb{P}\left[E_{t}\right]+\bar{V} \beta_{t}^{v} .
$$

This allows us to simplify expression (17) as

$$
\begin{aligned}
& \mathbb{E}\left[c \sum_{t=0}^{k-1}\left\|x_{t}\right\|^{2}\right]-V_{0} \\
& \leq \sum_{t=0}^{k-1}-c \mathbb{E}\left[\left\|x_{t}\right\|^{2} \mid E_{t}\right] \mathbb{P}\left[E_{t}\right]+\bar{V} \beta_{t}^{v}+c \mathbb{E}\left[\left\|x_{t}\right\|^{2}\right] \\
& \leq \sum_{t=0}^{k-1}-c \mathbb{E}\left[\left\|x_{t}\right\|^{2} \mid E_{t}\right] \mathbb{P}\left[E_{t}\right]+\bar{V} \beta_{t}^{v}+ \\
& =\sum_{t=0}^{k-1} \bar{V} \beta_{t}^{v}+c \mathbb{E}\left[\left\|x_{t}\right\|^{2} \mid \neg E_{t}\right] \mathbb{P}\left[\neg E_{t}\right] \\
& \leq \sum_{t=0}^{k-1} \beta_{t}^{v}\left(\bar{V}+c \mathbb{E}\left[\left\|x_{t}\right\|^{2} \mid \neg E_{t}\right]\right) .
\end{aligned}
$$

Since $x_{t} \in \mathcal{X}, t \in \mathbb{N}$, and $\mathcal{X}$ is a compact set containing the origin, there exists an $r \geq 0$ such that $\|x\|^{2} \leq r$. Therefore,

$$
\mathbb{E}\left[\sum_{t=0}^{k-1}\left\|x_{t}\right\|^{2}\right] \leq \frac{V_{0}}{c}+\left(\frac{\bar{V}}{c}+r\right) \sum_{t=0}^{k-1} \beta_{t}^{v},
$$

which is finite for all $k$, since $\left(\beta_{t}^{v}\right)_{t \in \mathbb{N}}$ is summable.

\section{Proof of Theorem IV.6.}

First, note that using the monotonicity of coherent risk measures [25, Sec. 6.3, (R2)], a straightforward inductive argument allows us to show that under Condition (i),

$$
\mathbf{T} \hat{V}_{N} \leq \hat{V}_{N}, \quad \forall N \in \mathbb{N} .
$$

Since $\overline{\mathcal{Z}} \times W \subseteq \operatorname{dom} \hat{V}_{N}$, recall that by definition (15), we have for any $(z, w) \in \overline{\mathcal{Z}} \times W$ that

$$
\begin{aligned}
& \hat{V}_{N}(z, w)=\ell\left(x, \hat{\kappa}_{N}(z, w), w\right) \\
& \quad+\rho_{w, s}^{\beta}\left[\hat{V}_{N-1}\left(\tilde{f}^{\hat{\kappa}_{N}}\left(z, w, w^{\prime}\right), w^{\prime}\right)\right] .
\end{aligned}
$$

Therefore, we may write

$$
\begin{aligned}
& \rho_{w, s}^{\beta}\left[\hat{V}_{N}\left(\tilde{f}^{\hat{\kappa}_{N}}\left(z, w, w^{\prime}\right), w^{\prime}\right)\right]-\hat{V}_{N}(z, w) \\
& =\rho_{w, s}^{\beta}\left[\hat{V}_{N}\left(\tilde{f}^{\hat{\kappa}_{N}}\left(z, w, w^{\prime}\right), w^{\prime}\right)\right]-\ell\left(x, \hat{\kappa}_{N}(z, w), w\right) \\
& \quad-\rho_{w, s}^{\beta}\left[\hat{V}_{N-1}\left(\tilde{f}^{\hat{\kappa}_{N}}\left(z, w, w^{\prime}\right), w^{\prime}\right)\right. \\
& \leq-\ell\left(x, \hat{\kappa}_{N}(z, w), w\right) \leq-c\|x\|^{2},
\end{aligned}
$$

where the first inequality follows by (18) and monotonicity of coherent risk measures. The second inequality follows from Condition (ii). Therefore, $V:(z, w) \rightarrow \hat{V}_{N}(z, w)+\delta_{\overline{\mathcal{Z}}}(z)$ satisfies the conditions of Lemma IV.5 and the statement follows. 